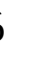
7

\title{
Evidence for a 'trap-and-flip' mechanism in a proton-dependent lipid transporter
}

Elisabeth Lambert ${ }^{1+}$, Ahmad Reza Mehdipour ${ }^{2 \dagger}$, Alexander Schmidt $^{3}$, Gerhard Hummer ${ }^{2,4}$, Camilo Perez ${ }^{1^{*}}$ 5

${ }^{1}$ Biozentrum, University of Basel, 4056 Basel, Switzerland

2Department of Theoretical Biophysics, Max Planck Institute of Biophysics, 60438 Frankfurt am Main, Germany

${ }^{3}$ Proteomics Core Facility, Biozentrum, University of Basel, Basel, Switzerland

${ }^{4}$ Institute of Biophysics, Goethe University Frankfurt, 60438 Frankfurt am Main, Germany 1

Abbreviated title: Trap-and-flip mechanism in a proton-dependent flippase

*Correspondence should be addressed to C.P. (email: camilo.perez@unibas.ch)

${ }^{\dagger}$ These authors contributed equally to this work 18 
Transport of lipids across membranes is fundamental for diverse biological pathways in cells. Multiple ion-coupled transporters participate in lipid translocation, but their mechanisms remain largely unknown. Major facilitator superfamily (MFS) lipid transporters play central roles in cell wall synthesis, brain development and function, lipids recycling, and cell signaling. Recent structures of MFS lipid transporters revealed overlapping architectural features pointing towards a common mechanism. Here we used cysteine disulfide trapping, molecular dynamics simulations, mutagenesis analysis, and transport assays in vitro and in vivo, to investigate the mechanism of LtaA, a proton-dependent MFS lipid transporter essential for lipoteichoic acids synthesis in the pathogen Staphylococcus aureus. We reveal that LtaA displays asymmetric lateral openings with distinct functional relevance and that cycling through outward- and inward-facing conformations is essential for transport activity. We demonstrate that while the entire amphipathic central cavity of LtaA contributes to lipid binding, its hydrophilic pocket dictates substrate specificity. We propose that LtaA catalyzes lipid translocation by a 'trap-and-flip' mechanism that might be shared among MFS lipid transporters.

Major facilitator superfamily (MFS) transporters are found in all kingdoms of life and move a large variety of molecules across biological membranes [1-8]. Structural characterization of MFS transporters that participate in the uptake of water-soluble molecules and extrusion of drugs has contributed to a broad understanding of their transport mechanism [4, 8-17]. However, multiple reports have attributed alternative functions to MFS transporters, such as translocation of lipids associated with fundamental biological pathways. Some examples include the bacterial lysophospholipid transporter LpIT, involved in lipids recycling in Gramnegative bacteria $[7,18]$; the human transporter MFSD2A, expressed at the blood-brain- and blood-retinal-barrier, contributing to major uptake of docosahexaenoic acid (DHA) [5, 6, 1921]; the human transporters Spns2 [22, 23], and MFSD2B [24], which contribute to transport of sphingosine 1-phosphate (S1P) in endothelial cells and erythrocytes; and the gentiobiosyldiacylglycerol transporter LtaA, involved in cell wall synthesis in Staphylococcus aureus [25, 26]. However, despite their well described cellular roles, the mechanisms of MFS lipid transporters remain insufficiently understood. 
nasopharynx of the human host [26-28]. LtaA takes part in the assembly of lipoteichoic acids, phosphate-rich polymers important for control of bacterial cell division, protection from environmental stress, host cell adhesion, antibiotic resistance, biofilm formation, and immune evasion [29-32]. S. aureus lipoteichoic acids display a polymer of 1,3-glycerol-phosphate repeat units attached to C-6 of the non-reducing glucosyl of the glycolipid gentiobiosyldiacylglycerol [31-33]. This glycolipid is synthesized at the cytoplasmic leaflet of the membrane by the glycosyltransferase YpfP, and is translocated to the outer leaflet by the activity of $\operatorname{LtaA}[25,26]$. The essential role of $\operatorname{LtaA}$ in adjusting the pool of glycolipids available at the extracellular side of the membrane, makes this protein a central player for lipoteichoic acids assembly and a potential target for drugs aiming to counteract antimicrobial resistant $S$. aureus strains e.g., methicillin-resistance $S$. aureus (MRSA) and vancomycin-resistant S. aureus (VRSA) [30].

Two different general models of transporter-catalyzed lipid translocation have been proposed in the past [34-42]. A 'trap-and-flip' model, in which the lipid substrate is retrieved from one leaflet of the membrane, enclosed into a central cavity, and then delivered to the other leaflet $[40,43,44]$, and a 'credit-card' model that departs from the classical alternatingaccess model and involves translocation of the lipid head-group across a hydrophilic cleft or cavity in the transport protein, while aliphatic chains remain embedded in the membrane [36$38,41,42,45,46]$. However, it is not known which of these two models describe better the mechanism of MFS lipid transporters. Answering this question is not only important to understand the basis of the processes catalyzed by these proteins, but could also provide a foundation for the design of drugs and/or lipid-linked-bioactive molecules targeting cells or organs expressing pharmacologically relevant proteins from this superfamily.

Until now, only the high-resolution structures of outward-facing LtaA and inwardfacing MFSD2A have been elucidated $[21,26]$. Both transporters displayed the canonical MFS fold of 12 transmembrane (TM) helices and an amphipathic central cavity that has not been observed in any MFS transporter of water-soluble molecules. The similar architectural features observed in the structures of LtaA and MFSD2A indicate common elements in their transport mechanisms and likely among all MFS lipid transporters. Here, we used cysteine disulfide trapping of outward- and inward-facing LtaA, in combination with molecular dynamics simulations, mutagenesis analysis, and transport assays in vitro and in vivo, and showed that cycling through outward- and inward-facing conformations is essential for LtaA 
activity. We demonstrate that LtaA displays membrane exposed lateral openings with distinct functional relevance and characterized the architecture and biochemical properties of the amphipathic central cavity during alternating-access. Our results indicate that while the hydrophilic pocket of the amphipathic central cavity dictates substrate specificity, the hydrophobic pocket is only relevant for aliphatic chains binding. We describe critical mechanistic elements revealing that LtaA adopts a 'trap-and-flip' mechanism that might be shared among MFS lipid transporters.

\section{$\underline{\text { Results }}$}

\section{Model of inward-facing LtaA and validation by cysteine cross-linking}

To investigate whether LtaA uses a 'trap-and-flip' or a 'credit-card' mechanism, we first aimed to establish a system that allowed us to perform cysteine disulfide trapping of endpoint conformations of LtaA during its transport cycle. The architecture of the previously solved structure of LtaA [26], facilitates cysteine disulfide trapping of outward-facing states, whereas there is no structural information to guide trapping of inward-facing states. Thus, we

111 first generated an inward-facing model of LtaA using 'repeat-swap' modeling [47]. Like other 112 transporters from the MFS superfamily, the topology of LtaA consists of two domains, a N113 terminal domain (TM1-TM6; domain-1), and a C-terminal domain (TM7-TM12; domain-2), 114 each of which contains two structural repeats with inverted-topology related by a pseudo115 rotational two-fold symmetry axis parallel to the plane of the membrane (Fig. 1A,B). After 116 swapping the conformations of the inverted repeats observed in the outward-facing structure 117 of LtaA (PDB ID 6S7V) [26, 47], we constructed a large set of models in silico that were refined 118 aiming to improve side chains packing, stereochemistry, and modeling scores. The models 119 with the best scores converged to one conformation (Fig. 1C and Suppl. Table. 1), which 120 displayed multiple interactions between the extracellular parts of TM1-TM7, TM2-TM11, and

121 TM5-TM8, sealing the entrance to the central cavity (Fig. 1C). In contrast, the cytoplasmic 122 regions of helices TM2-TM11, TM5-TM8, and TM4-TM10, lining the entrance to the central 123 cavity from the cytoplasm, are away from each other about $16.0 \pm 0.1 \AA, 16.0 \pm 0.1 \AA$, and

$12417.6 \pm 0.2 \AA$, respectively (Fig. 1C). The helical loop between TM6 and TM7 that connects the $125 \mathrm{~N}$ - and C-terminal domains was modeled based on the conformation observed in the outward126 facing structure. 
To validate the inward-facing model, we selected pairs of residues among the 128 extracellular regions for which $C \beta-C \beta$ distances were less than $7 \AA$, but which present $C \beta-C \beta$ 129 distances of over $12 \AA$ in the outward-facing structure (Suppl. Fig. 1). Based on these criteria, 130 we identified the pairs F45-T253, A53-T366, and K166-I250 (Fig. 2A and Suppl. Fig. 1), 131 occupying three different positions that provide good coverage of the conformational change 132 predicted by our models. We then introduced cysteine residues at these positions on a starting 133 construct in which the one native cysteine in LtaA was replaced with serine. The cysteine-less 134 LtaA variant effectively performed glycolipid flipping in proteoliposomes (Suppl. Fig. 2). The 135 three mutants F45C-T253C, A53C-T366C, and K166C-I250C were then irreversibly crosslinked 136 with N,N'-(o-phenylene)-dimaleimide (o-PDM), which has a spacer arm length of $6 \AA$. 137 Crosslinked and non-crosslinked LtaA mutants were digested with either trypsin or 138 chymotrypsin, and analyzed by high-resolution liquid chromatography-mass spectrometry 139 (LC-MS) to evaluate the presence of non-crosslinked cysteine containing peptides. The 140 peptides abundance was normalized against an internal reference peptide. We successfully 141 identified non-crosslinked peptides in untreated samples of the three mutants F45C-T253C, A53C-T366C, and K166C-I250C (Fig. 2A and Suppl. Fig. 3). The abundance of these peptides was clearly diminished in the crosslinked protein samples (Fig. 2A), demonstrating that the selected pairs of residues are in close proximity as predicted in the inward-facing model. shown to interact at the cytoplasmic region of the outward-facing structure (Fig. 2B). Thus, we introduced cysteine residues at the positions K80-E339 and K141-N276, present at the cytoplasmic ends of TM2-TM11 and TM5-TM8, respectively. $C \beta-C \beta$ distances between these residues are smaller than $7.5 \AA$ in the outward-facing structure, but larger than $12 \AA$ in the inward-facing models (Suppl. Fig. 1). LC-MS analysis of the double mutants K80C-E339C and

151 K141C-N276C confirmed the proximity of these residues as non-crosslinked peptides are more 152 abundant in untreated samples, whereas in the presence of the cross-linking agent their 153 abundances decrease substantially (Fig. 2B). In summary, our cross-linking analysis support 154 the predicted conformation and interactions reported by the inward-facing model of LtaA and 155 indicate the position of residues to guide cysteine disulfide trapping of LtaA conformations. 


\section{Alternating conformations in proteoliposome membranes}

We investigated the conformations displayed by LtaA in membranes by evaluating the cross-linking of double cysteine mutants reconstituted in proteoliposomes (Fig. 2C). The cysteine pairs reported on the conformation of the TM helices that line the lateral openings, TM2-TM11 and TM5-TM8 (Fig. 1C). We screened for successful cross-links by using a gel-shift assay in which we first incubated with the o-PDM cross-linker, followed by treating the proteoliposomes with 5-kDa PEG-maleimide (mPEG5k) [48]. This treatment generates a substantial shift in the protein mobility in polyacrylamide gel electrophoresis as mPEG5k irreversibly binds free cysteines. However, if the introduced cysteines are cross-linked by oPDM, then they will not react with mPEG5k and no shift in gel mobility would be observed. We evaluated the cross-linking of residues A53C-T366C (TM2-TM11) and K166C-I250C (TM5TM8), positioned at the extracellular region, and K80C-E339C (TM2-TM11) and K141C-N276C (TM5-TM8), located at the cytoplasmic region (Fig. 2C and Suppl. Fig. 4A,B). Before crosslinking, each double-cysteine mutant showed a gel shift after incubation with mPEG5K, thus demonstrating PEGylation of free cysteines (Fig. 2C and Suppl. Fig. 4C), albeit the shift of K166C-I250C is less prominent (Fig. 2C). In contrast, after treatment with o-PDM, all the double cysteine mutants were protected from PEGylation, thus showing that all mutants were successfully cross-linked. The cysteine-less control LtaA, did not show a gel shift in any of the conditions (Fig. 2C and Suppl. Fig. 4C), demonstrating that the shifts observed for the mutants were due to PEGylation of cysteines. These results support that when embedded in the membrane of proteoliposomes, LtaA can adopt conformations where residues at the lateral openings lined by TM2-TM11 and TM5-TM8 display similar distances to those reported by the outward-facing structure and the inward-facing model.

\section{Alternating-access to the central cavity is essential for function}

The cross-linking results described above showed that LtaA can cycle through outwardand inward-facing conformations. However, it is not known whether this is required for function. This is important because some flippases and scramblases use a 'credit card' mode of transport, where exposing a side cleft or a cavity to one side of the membrane is sufficient for catalysis of lipid transport across the membrane [36-38, 41, 49]. To answer whether alternating between inward- and outward-facing conformations is important for LtaA activity, we performed copper chloride catalyzed cross-linking of residues located at the lateral 
openings lined by TM2-TM11 and TM5-TM8, and determined proton-coupled glycolipid

192 transport activity of cross-linked LtaA variants in proteoliposomes (Fig. 3). In this assay, the

193 addition of the $\mathrm{K}^{+}$-selective ionophore valinomycin generates a membrane potential of about

$194-60 \mathrm{mV}$, which drives proton influx. Acidification of the lumen of proteoliposomes quenches

195 the fluorophore 9-amino-6-chloro-2-methoxyacridine (ACMA) causing a decrease in the

196 fluorescence [26]. The double-cysteine mutants A53C-T366C (TM2-TM11) and K166C-I250C

197 (TM5-TM8) close the extracellular side openings, whereas the mutants K80C-E339C (TM2-

198 TM11) and K141C-N276C (TM5-TM8) close the cytoplasmic openings (Fig. 3A and Suppl. Fig.

199 4A,B). Our results show that except for K141C-N276C (TM5-TM8), independent cross-linking

200 of the remaining three lateral openings decreases LtaA activity relative to non-cross-linked

201 mutants (Fig. 3A).

202 In addition, we aimed to completely close the cytoplasmic or extracellular cavities and

203 test the effect on LtaA activity (Fig. 3B). To do this, we constructed the mutant A53C-T366C-

204 K166C-I250C that after cross-linking would close the extracellular pathway, while the mutant

205 K80C-E339C-K141C-N276C would close the cytoplasmic pathway (Fig. 3B and Suppl. Fig. 4A,B).

206 Our results show that in contrast to non-cross-linked proteins, both mutants display

207 background quenching levels, similar to that observed for protein-free liposomes, thus,

208 indicating strong inhibition of transport activity (Fig. 3B). We confirmed cross-linking of each

209 double- and tetra-cysteine mutant reconstituted in proteoliposomes by gel shift assays after

210 incubation with mPEG5K (Fig. 3C,D and Suppl. Fig. 4D), which showed that after treatment

211 with copper chloride, all the mutants were protected from PEGylation, whereas before cross-

212 linking a gel shift was observed. This confirmed that all mutants were successfully cross-linked

213 in the proteoliposomes samples used in the assay.

214 Taking together, these results reveal that alternating opening to both sides of the 215 membrane is a requirement for LtaA function. However, not all lateral openings seem to have 216 the same functional relevance. In particular, our results demonstrate that while both extracellular lateral openings are similarly important for function, the cytoplasmic lateral

218 opening lined by TM2 and TM11 has a more significant role, as revealed by the low activity of 219 the cross-linked variant K80C-E339C. In contrast, cross-linking of the cytoplasmic lateral opening lined by TM5 and TM8, does not seem to affect LtaA function strongly. 


\section{Dynamics of lateral openings}

224 For lipid transporters that adopt a 'trap-and-flip' mechanism, substrate binding and 225 release involve movement of lipids through lateral openings of the translocation channel [18, $22621,40,43,44]$. We studied the dynamic behavior of the lateral openings in LtaA when the 227 protein is embedded in a lipid bilayer. To do this, we performed molecular dynamics (MD) 228 simulations of outward- and inward-facing LtaA in a membrane composed of POPG (65\%), diacylglycerol (20\%), cardiolipin (10\%), and gentiobiosyl-diacylglycerol (5\%), resembling the membrane of $S$. aureus [50]. During the simulations both states were found to be stable as judged by RMSD plots (Suppl. Fig. 5). The simulations revealed that all the optimized inwardfacing models exhibit a cavity which is open to the cytoplasm and closed to the extracellular space, whereas the cavity of the outward-facing state is open to the extracellular space and closed to the cytoplasm (Fig. 4A). In agreement with the observed wider opening of the extracellular lateral openings, the simulations of outward-facing LtaA showed the intrusion of glycolipid and POPG molecules into the putative translocation pathway (Fig. 4C). The glycolipid was seen to intrude from the TM5-TM8 opening, with one of the aliphatic tails reaching to the C-terminal hydrophobic pocket, whereas two POPG molecules intrude from the TM2-TM11 opening (Fig. 4C). By contrast, simulations of inward-facing LtaA did not show lipids entering the cytoplasmic cavity.

In the MD simulations of the inward-facing conformation, we found that the cytoplasmic opening between TM2 and TM11 is wider and more dynamic than that between TM5 and TM8 (Fig. 4B). The lateral opening between TM2 and TM11 is thus a likely route for entry of glycolipids into the substrate binding site of the transporter. By contrast, in the outward-facing conformation, the two gates have similar widths and dynamics (Fig. 4B). Together with the results from the cysteine trapping analysis, this data suggests that both extracellular openings are a likely route for glycolipids exit, whereas the cytoplasmic lateral opening lined by TM2 and TM11 is more relevant for function as it may provide more room for glycolipids to enter the translocation pathway.

\section{The hydrophobic pocket is relevant for lipid transport}

Inspection of the central cavity in the in silico analysis shows that similarly to what was observed in the outward-facing crystal structure of LtaA [26], the central cavity of the inward-

254 facing state is amphipathic (Fig. 5A). The cavity displays a hydrophilic pocket, enclosed mainly 
by residues from the N-terminal domain (E32, R35, D68, W127 and W150), which we have previously shown to be relevant for recognition of the glycolipid headgroup [26], and a hydrophobic pocket, enclosed mainly by residues from the C-terminal domain (V234, L237, 258 C263, L296, L300, L309, 1316, and Y320) (Fig. 5A). The recent structure of the MFSD2A transporter trapped in an inward-facing conformation, displays a similar amphipathic central cavity (Suppl. Fig. 6) [21]. Computational docking of a glycolipid molecule to inward-facing LtaA suggests that the gentiobiosyl headgroup is preferentially accommodated in the hydrophilic pocket, whereas the diacylglycerol aliphatic tails are docked into the hydrophobic pocket (Fig. 5B).

A striking feature of the central cavity observed in LtaA and MFSD2A [21], and to our knowledge, not observed in other MFS structures available to date, is the presence of the highly hydrophobic pocket at the C-terminal domains of these transporters (Fig. 5A and Suppl.

Fig. 6). To test the importance of this pocket in LtaA, we have designed mutants that introduce polar residues, thus making it more hydrophilic. We then evaluated growth of $S$. aureus NCTC8325 $\Delta$ ItaA cells complemented with ectopic copies of the ItaA gene carrying these mutations (Fig. 5C and Suppl. Fig 7). The variants V234T/L237N/I297S, C263S/L309Q/I316N, and Y320R display marked growth defects, whereas the mutant L296D/I316N do not affect growth. Each mutant was also purified and reconstituted into proteoliposomes, followed by determination of their flipping activity (Fig. 5D). In agreement with the results from S. aureus growth assays, the mutants V234T/L237N/I297S, C263S/L3090/I316N, and Y320R display low relative activity compared to LtaA-WT (Fig. 5D), whereas L296D/I316N display the highest activity among all mutants. In contrast, introducing a mutation that scarcely increases the polarity of the cavity but that changes the size of residues V234 and 1316, displayed low relative flipping activity compared to LtaA-WT, but does not affect growth of S. aureus NCTC8325 $\Delta$ ltaA cells (Fig. 5C,D and Suppl. Fig 7). Taken together, these results support a fundamental role of the hydrophobic pocket in glycolipid transport. As suggested by docking analysis, it is likely that this pocket is involved in binding of the aliphatic tails of the glycolipid substrate. The striking hydrophobicity of the C-terminal TM helices 7, 8, and 10 in multiple MFS lipid transporters (Suppl. Fig. 8), and the involvement in coordination of the aliphatic chain of lysophospholipid as revealed by the structure of MFSD2A [21], suggest a shared mechanistic role of the hydrophobic pocket in lipid-tails binding in MFS lipid transporters. 
The hydrophilic pocket dictates substrate specificity

So far, our results suggest that during transport, LtaA encloses the full glycolipid substrate in the amphipathic cavity. However, understanding the relevance of the individual parts of the substrate molecule, headgroup and aliphatic chains, is fundamental for future

291 design of pharmacologically relevant molecules targeting this and other MFS lipid transporters.

292 To gain insight into whether LtaA displays higher selectivity towards the headgroup than for 293 the diacylglycerol moiety, we performed flipping assays with LtaA-WT co-reconstituted in 294 proteoliposomes together with NBD-labeled Glc2-DAG (gentiobiosyl-diacylglycerol) and 295 increasing concentrations of Gal $_{2}$-DAG (digalactosyl-diacylglycerol) (Fig. 5E). Glucose and galactose differ only in the orientation of the $-\mathrm{OH}$ group at the C-4 position. Thus, we 297 hypothesized that if the headgroup is more relevant for substrate recognition than the 298 aliphatic chains, then transport of $\mathrm{Gl}_{2}$-DAG-NBD will be not be affected, since the difference between glucose and galactose would preclude $\mathrm{Gal}_{2}$-DAG from being a good competitor. On the other hand, if the diacylglycerol moiety is more relevant for substrate recognition, we expect $\mathrm{Gal}_{2}$-DAG to be a strong competitor, thus, resulting in marked decrease of Glc2-DAGNBD transport. Our results show that even under a high excess of $\mathrm{Gal}_{2}-\mathrm{DAG}$, there is no significant effect on Glc $_{2}$-DAG-NBD transport (Fig. 5E). We have previously shown that gentiobiose ( $\beta$-D-Glc-(1,6)-D-Glc), a disaccharide with the same composition and conformation as the glycolipid headgroup ( $\left.\mathrm{Glc}_{2}-\mathrm{DAG}\right)$, inhibits lipid transport [26]. Taken together, these results suggest that an intact headgroup is highly relevant for substrate binding and transport, and that even small changes to the headgroup abolishes recognition. Independent of the presence of the diacylglycerol moiety and its predicted binding to the hydrophobic pocket, the headgroup seems to dictate whether a glycolipid can be a substrate

\section{Discussion}

Several transporters of the MFS superfamily have been structurally characterized in

314 one or multiple conformational states [4, 8-17] However, except for the outward-facing 315 structure of LtaA [26], solved by X-ray crystallography, and the inward-facing structure of 316 MFSD2A [21], solved by single particle cryo-electron microscopy, there are no additional 317 structures available of MFS lipid transporters. Despite the differences among their lipid 318 substrates, the distinct composition of bacterial and eukaryotic membranes, and their 
opposite vectorial lipid transport directions, LtaA and MFSD2A share multiple architectural similarities, including a canonical MFS fold of 12 TM helices and an amphipathic central cavity with asymmetric distribution of hydrophobic and hydrophilic residues (Suppl. Fig. 6). A similar arrangement of central cavity residues has been predicted to be present in the bacterial 323 Iysophospholipid transporter LpIT [18], and are likely to be part of the architecture of other MFS lipid transporters (Suppl. Fig. 8). These characteristics suggest a common mechanism of substrate recognition and translocation among these proteins. Indeed, LtaA and MFSD2A 326 display strong selectivity towards the headgroup of their lipid substrates $[6,26]$. In the case of MFSD2A the zwitterionic charge of the phosphatidylcholine headgroup is fundamental for ligand transport, whereas LtaA displays strong selectivity towards the gentiobiosyl disaccharide headgroup of the glycolipid. Furthermore, LtaA selects against an isomer of the disaccharide headgroup as shown by the poor competition displayed by digalactosyldiacylglycerol in transport assays (Fig. 5E). In contrast, LpIT has been shown to exhibit a more relaxed specificity towards the lipid headgroup, being able to transport lysophosphatidylethanolamine and lysophosphatidylglycerol lipids [18].

Although MFSD2A and LpIT have been shown to strongly select for lysophospholipids,

they display relaxed selectivity towards the length of the aliphatic chains $[6,18]$. MFSD2A transports docosahexaenoic acid (DHA), an essential omega-3 fatty acid for brain growth and cognitive function, in the form of lysophosphatidylcholine, but can also transport other lipids with at least 14-carbons acyl chain [6]. It is noteworthy that S. aureus membranes are rich in diacylglycerols with chains length ranging from $C_{15}$ to $C_{18}$, with the most dominant lipid species having a $\mathrm{C}_{17}: \mathrm{C}_{15}$ composition [25]. This variability among diacylglycerols in $\mathrm{S}$. aureus, and the measurable translocation of Glc 2 -DAG-NBD [26], which has a $\mathrm{C}_{10}$ acyl chain length and an NBD group linked to one of the diacylglycerol chains, suggest that LtaA displays similar relaxed specificity towards the length of the lipid part.

Our results strongly suggest that in contrast to mechanisms proposed for other lipid transporters, LtaA transports gentiobiosyl-diacylglycerol by a 'trap-and-flip' mechanism, which follows the classical alternating-access model of transport [51], with the entire 347 glycolipid entering and leaving the central translocation pathway (Fig. 5F). Inward-facing LtaA 348 binds a glycolipid molecule which enters through the lateral opening lined by TM2 and TM11, as suggested by the cross-linking analysis and MD simulations. This triggers a conformational 
presumably through any of the two extracellular lateral openings. Protonation of residues in the hydrophilic pocket allows transition to the inward-facing conformation, followed by proton release to the cytoplasm.

Similar to the asymmetric opening of extracellular and cytoplasmic cavities described here for LtaA, MFSD2A exhibits a wider opening of its cytoplasmic lateral openings [21]. In this case, the extracellular opening is predicted to be narrow due to the constriction imposed by a disulfide bond at the extracellular side of the transporter [21]. Our results suggest that LtaA exhibit wide open extracellular lateral openings, whereas the cytoplasmic openings are narrower. The distinct constrictions of the cavities that recruit the lipid substrate, cytoplasmic cavity in LtaA and extracellular cavity in MFSD2A, might be part of a selectivity filter that confers substrate specificity.

In summary, our results provide insights into the molecular mechanism of glycolipid transport by LtaA and support a 'trap-and-flip' model where asymmetrically open lateral 'gates' and extracellular and cytoplasmic cavities play an essential role. Our data suggests that the highly selective hydrophilic pocket dictates substrate specificity, but that the hydrophobic pocket is fundamental for aliphatic chains transport. The mechanistic elements described here might be shared by other MFS lipid transporters and can be decisive for the design of drugs targeting these proteins.

\section{Methods}

371 LtaA expression and purification. The gene encoding S. aureus LtaA was cloned into a modified pET-19b vector (Novagen), with an N-terminal His 10 affinity tag. LtaA WT and mutants were expressed in E. coli BL21 Gold (DE3) (Stratagene) cells. Cells were grown in

374 Terrific Broth (TB) medium supplemented with $1 \%$ glucose $(w / v)$ at 3709 . Overexpression was induced with $0.2 \mathrm{mM}$ Isopropyl $\beta$-D-1-thiogalactopyranoside (IPTG) for $1 \mathrm{~h}$. All following steps were performed at $4 \stackrel{\circ}{\circ}$, unless different specified. Cells were harvested by centrifugation, resuspended in 50mM Tris- $\mathrm{HCl}$, $\mathrm{pH} 8.0 ; 500 \mathrm{mM} \mathrm{NaCl} ; 5 \mathrm{mM} \beta$-mercaptoethanol; 0.5mM PMSF and disrupted in a M-110L microfluidizer (Microfluidics) at 10000 psi chamber pressure. Membranes were pelleted by ultracentrifugation and solubilized in $50 \mathrm{mM}$ Tris- $\mathrm{HCl}, \mathrm{pH} 8.0$;

$380200 \mathrm{mM} \mathrm{NaCl} ; 20 \mathrm{mM}$ Imidazole; $15 \%$ glycerol (v/v); $5 \mathrm{mM} \beta$-mercaptoethanol; $1 \%$ Lauryl 381 Maltose Neopentyl Glycol (w/v) (LMNG, Anatrace); 1\% N-dodecyl- $\beta$-D-maltopyranoside (w/v) 382 (DDM, Anatrace). After removing debri, the supernatant was loaded onto a pre-equilibrated 
NiNTA superflow affinity column (Qiagen). The column was washed with 50mM Tris- $\mathrm{HCl}, \mathrm{pH}$

8.0; $200 \mathrm{mM} \mathrm{NaCl} ; 50 \mathrm{mM}$ Imidazole; $10 \%$ glycerol (v/v); $5 \mathrm{mM} \beta$-mercaptoethanol; $0.02 \%$ LMNG and $0.02 \%$ DDM and then further washed with the same buffer only containing $0.02 \%$ LMNG. Elution was performed in the same buffer containing $200 \mathrm{mM}$ Imidazole. Buffer exchange to buffer $10 \mathrm{mM}$ Tris- $\mathrm{HCl} \mathrm{pH} 8.0 ; 150 \mathrm{mM} \mathrm{NaCl} ; 0.02 \%$ LMNG was performed using PD-10 columns (GE Healthcare). Analytical size exclusion chromatography was performed on a Superdex 10/300 GL column (GE Healthcare) in buffer $10 \mathrm{mM}$ Tris- $\mathrm{HCl}$, pH 8.0; $150 \mathrm{mM} \mathrm{NaCl}$; $0.02 \%$ LMNG. [52]

Mutagenesis. LtaA mutants were generated using overlap Extension-PCR, followed by DpnI digestion for two hours at $37^{\circ} \mathrm{C}$, and transformation into $E$. coli $\mathrm{DH} 5 \mathrm{a}$ cells. The mutations were confirmed by DNA sequencing (Microsynth). All oligos used for mutagenesis are listed in Suppl. table 2.

YpfP expression and purification. The gene encoding S. aureus YpfP was cloned into a modified pET-19b vector (Novagen) with an N-terminal His 10 affinity tag. YpfP was overexpressed in BL-21 Gold (DE3) (Stratagene) cells. Cells were grown in TB medium supplemented with $1 \%$ glucose $(w / v)$ at $37 \stackrel{\circ}{ }=$ until a cell density of $O_{600}=3$. Subsequently, cells were induced with $0.2 \mathrm{mM}$ IPTG for $16 \mathrm{~h}$ at $24 \stackrel{\circ}{\circ}$. Cells were harvested by centrifugation and resuspended in buffer $\mathrm{A}(50 \mathrm{mM}$ Tris- $\mathrm{HCl} \mathrm{pH} 8.0 ; 200 \mathrm{mM} \mathrm{NaCl} ; 3 \%$ glycerol; $3 \mathrm{mM} \beta$ mercaptoethanol) plus 0.5mM PMSF. Cells were disrupted using a tip sonication. After differential centrifugation, the supernatant containing YpfP was incubated with NiNTA resin and left stirring for $1 \mathrm{~h}$ at $4 \stackrel{\circ}{\circ} \mathrm{C}$. Washing was performed with buffer $\mathrm{A}$ complemented with 50 $\mathrm{mM}$ imidazole $\mathrm{pH}$ 8.0, followed by elution with buffer A complemented with $200 \mathrm{mM}$ imidazole pH8.0. YpfP was desalted in buffer $50 \mathrm{mM}$ Tris- $\mathrm{HCl} \mathrm{pH} 8.0 ; 200 \mathrm{mM} \mathrm{NaCl} ; 10 \%$ glycerol using PD-10 columns (GE healthcare). If required YpfP was concentrated using a

409 Vivaspin $2030 \mathrm{MWCO}$ until $2.4 \mathrm{mg} / \mathrm{ml}$, flash frozen in liquid nitrogen and stored at -80 ㄷ $\mathrm{C}$ until 410 further use.

412 Synthesis of NBD-glycolipid and glycolipid. Synthesis of glycolipd and nitrobenzoxadiazole 413 (NBD)-labelled glycolipid was performed using a modification of the protocol described by 414 Jorasch et al [53] and Kiriukhin et al [54]. A final concentration of 2mM UDP-Glucose (Sigma), 
2mM NBD-decanoyl-2-decanoyl-sn-Glycerol (Cayman), and $1.2 \mathrm{mg} / \mathrm{ml}$ purified YpfP were incubated together for $16 \mathrm{~h}$ at $30^{\circ} \mathrm{C}$. The reaction product was separated using thin-layer chromatography (TLC) with a silica gel matrix (Sigma) in a solvent mixture consisting of chloroform:methanol:water (65:25:4, vol/vol/vol). Silica containing the NBD-glycolipid was recovered from plates, and the NBD-glycolipid was extracted from the silica by incubation with a solvent mixture of chloroform:methanol (50:50, vol/vol), followed by drying of the anchor-

421 LLD under argon atmosphere, and subsequently resuspension in $20 \mathrm{mM}$ Tris- $\mathrm{HCl} \mathrm{pH} 8.0 ; 150$ $\mathrm{mM} \mathrm{NaCl}$. NBD-glycolipid was flash frozen in liquid nitrogen, and stored at $-80^{\circ} \mathrm{C}$ until further use. Reaction products were previously characterized [26]. Non-labelled glycolipid was prepared similarly by incubation of $2 \mathrm{mM}$ UDP-Glucose, $2 \mathrm{mM}$ 1,2-dimyristoyl-sn-glycerol (Avanti) and $1.2 \mathrm{mg} / \mathrm{ml} \mathrm{YpfP} \mathrm{for} 16 \mathrm{~h}$ at $30^{\circ} \mathrm{C}$.

Formation of LtaA proteoliposomes. LtaA was reconstituted in unilamellar liposomes prepared by extrusion through polycarbonate filters (400 $\mathrm{nm}$ pore size) from a $3: 1(\mathrm{w} / \mathrm{w}$ ) mixture of E. coli polar lipids and L- $\alpha$-phosphatidylcholine (Avanti polar lipids) resuspended in $20 \mathrm{mM}$ Tris- $\mathrm{HCl} \mathrm{pH} \mathrm{8.0;150mM} \mathrm{NaCl}$ and $2 \mathrm{mM} \beta$-mercaptoethanol. After saturation with DDM (Anatrace), liposomes were mixed with purified LtaA in a 50:1 (w/w) lipids/protein ratio. DDM was removed after incubation with BioBeads (BioRad). Proteoliposomes were centrifugated, washed and resuspended to a final concentration of $20 \mathrm{mg} / \mathrm{ml}$ lipids; $7.8 \mu \mathrm{M}$ LtaA. The proteoliposomes were flash-frozen in liquid nitrogen and stored at $-80^{\circ} \mathrm{C}$ until further use.

In vitro flipping assay. Before performing flipping assays, proteoliposomes were thawed, their resuspension buffer was exchanged to $20 \mathrm{mM} \mathrm{MES} \mathrm{pH} \mathrm{6.5;} 150 \mathrm{mM} \mathrm{NaCl}$, and the product of the NBD-glycolipid synthesis reaction was incorporated by performing freeze/thaw cycles. Proteoliposomes and protein-free liposomes were diluted to a concentration of $2 \mathrm{mg} / \mathrm{ml}$ lipids followed by extrusion through polycarbonate filters ( $400 \mathrm{~nm}$ pore size). Proteoliposomes were immediately used for flipping assays. In case of competition assays with digalactosyldiacylglycerol (DGDG). DGDG powder (Avanti) was resuspended in $20 \mathrm{mM}$ Tris$\mathrm{HCl} ; 150 \mathrm{mM} \mathrm{NaCl}$ and incorporated into proteoliposomes during freeze/thaw cycles together with the NBD-glycolipid. Flipping of NBD-glycolipid was assessed by determining the

446 (Sigma) after 200 seconds of starting fluorescence recording. 100 seconds before finishing 
data recording, $0.5 \%$ Triton X100 was added to permeabilize the liposomes, making all NBDglycolipid molecules accessible to dithionite reduction. The fluorescence after Triton X100 addition was used for baseline calculations. Fluorescence was recorded at $20^{\circ} \mathrm{C}$ using a Jasco

450 Fluorimeter. The excitation and emission wavelengths were 470 and $535 \mathrm{~nm}$, respectively. For 451 analysis the fluorescence intensity was normalized to $F / F_{\max }$. Relative flipping activities were 452 calculated as follows: relative activity $=100 \times\left(\left(F / F_{\max }\right)_{i}-\left(F / F_{\max }\right)_{\text {liposomes }}\right) /\left(\left(F / F_{\max }\right)_{w t}-\right.$ $\left.453\left(F / F_{\max }\right)_{\text {liposomes }}\right)$, where $\mathrm{i}$ corresponds to each respective treatment/mutants, liposomes 454 corresponds to liposomes without protein, wt corresponds to wild type LtaA and F/Fmax values correspond to the normalized fluorescence values at the plateau after addition of sodium dithionite. Curves were plotted using GraphPad Prism 8. Time courses of the dithionite-induced fluorescence decay in liposomes were repeated at least 3 times for each individual experiment.

Proton-transport assay. LtaA proteoliposomes and protein-free liposomes were thawed, and their internal buffer exchanged to $5 \mathrm{mM}$ HEPES pH7.3; $100 \mathrm{mM} \mathrm{KCl}$. Glycolipid was incorporated during freeze/thaw cycles followed by extrusion through polycarbonate filters (400 $\mathrm{nm}$ pore size). After 90 s of sonication, proteoliposomes and protein-free liposomes were diluted 25-fold in buffer containing $5 \mathrm{mM} \mathrm{HEPES} \mathrm{pH} \mathrm{7.3;} 10 \mathrm{mM} \mathrm{KCl} ; 90 \mathrm{mM} \mathrm{NaCl} ; 0.5 \mu \mathrm{M}$ 9amino-6-chloro-2-methoxyacridine (ACMA). Fluorescence was recorded using a Jasco Fluorimeter with excitation and emission wavelengths of 410 and $480 \mathrm{~nm}$ respectively. When the fluorescence signal was stable, $\mathrm{H}^{+}$influx was initiated by establishing a membrane potential by the addition of the potassium ionophore valinomycin ( $5 \mathrm{nM})$. Time courses of the proton-transport assay in proteoliposomes were repeated at least 3 times for each individual experiment. Crosslinking was performed before the measurement by addition of $2 \mathrm{mM} \mathrm{CuCl} 2$ to the proteoliposomes during the buffer exchange and incorporation of glycolipid steps. After $1 \mathrm{~h}$ incubation at $\mathrm{RT}$ in the dark, $\mathrm{CuCl}_{2}$ was removed by centrifugation, and proteoliposomes were resuspended in buffer 5 mM HEPES, pH7.3; 100mM KCl.

LtaA crosslinking and PEGylation. LtaA mutants incorporated into proteoliposomes were incubated with $2 \mathrm{mM} \mathrm{CuCl}_{2}$ or N,N'-1,2-phenylenedimaleimide (o-PDM) for $1 \mathrm{~h}$ at $\mathrm{RT}$ in the dark. In case of non-crosslinked samples, proteoliposomes were incubated with a proportional volume of DMSO or buffer. Crosslinkers were removed by centrifugation and washing with 
buffer. To PEGylate free cysteines, LtaA mutants were incubated for $3 \mathrm{~h}$ at RT in the presence of $0.5 \mathrm{mM}$ mPEG5K-Maleimide (Sigma) and 0.5\% SDS. Proteins were separated on 15\% polyacrylamide gels and visualized with QuickBlue Protein stain (Lubio science).

Sample preparation for LC-MS analysis. LtaA mutants were purified as described above, and concentrated to a concentration of $0.6 \mathrm{mg} / \mathrm{ml}$. Purified LtaA was incubated for $1 \mathrm{~h}$ at RT in the dark in the absence or presence of $2 \mathrm{mM}$ o-PDM. Afterwards, $10 \mathrm{mM} \beta$-mercatoethanol was added to quench the crosslinker. 1-2 ug of either crosslinked or non-crosslinked LtaA protein were dissolved in $20 \mu$ digestion buffer $(0.02 \%$ of LMNG; $1 \mathrm{M}$ urea; $0.1 \mathrm{M}$ ammoniumbicarbonate; $10 \mathrm{mM}$ tris(2-carboxyethyl) phosphine (TCEP); $15 \mathrm{mM}$ chloroacetamide, $\mathrm{pH}=8.5)$, reduced and alkylated for $1 \mathrm{~h}$ at $37^{\circ} \mathrm{C}$. Proteins were digested by incubation with either sequencing-grade modified trypsin (1/50, w/w; Promega, Madison, Wisconsin), chymotrypsin sequencing grade (1/50, w/w, Sigma-Aldrich) or lys-C (1/100, w/w, Wako) overnight at $37^{\circ} \mathrm{C}$. Then, the peptides were cleaned using iST cartridges (PreOmics, Munich) according to the manufacturer instructions. Samples were dried under vacuum and dissolved in $0.1 \%$ formic acid solution at $0.5 \mathrm{pmol} / \mathrm{ul}$. All samples were prepared in triplicates.

Label-free targeted PRM-LC-MS analysis of cysteine-containing peptides. In a first step, parallel reaction-monitoring (PRM) assays [55] were generated for all the peptides of LtaA WT and the peptides of the 5 different LtaA cysteine mutants, for each protease. These peptides include the reference peptide for normalization, that is shared for all mutants. Therefore, the specific peptide sequences were loaded into Skyline (version 20.2.0.343 (https://brendanxuw1.gs.washington.edu/labkey/project/home/software/Skyline/begin.view) and transitions were predicted using the integrated PROSIT algorithm for double and triple charged precursors. Then, protease and isoform specific isolation mass lists were exported and used

504 to generate specific targeted LC-MS analyses. This analysis was carried as described previously

505 [56]. Chromatographic separation of peptides was carried out using an EASY nano-LC 1000 system (Thermo Fisher Scientific), equipped with a heated RP-HPLC column $(75 \mu \mathrm{m} \times 30 \mathrm{~cm})$ 507 packed in-house with $1.9 \mu \mathrm{m} \mathrm{C18} \mathrm{resin} \mathrm{(Reprosil-AQ} \mathrm{Pur,} \mathrm{Dr.} \mathrm{Maisch).} \mathrm{Aliquots} \mathrm{of} 1$ pmol total peptides were analyzed per LC-MS/MS run using a linear gradient ranging from $95 \%$ solvent $\mathrm{A}$

509 ( $0.15 \%$ formic acid, $2 \%$ acetonitrile) and 5\% solvent B (98\% acetonitrile, $2 \%$ water, $0.15 \%$ 510 formic acid) to $30 \%$ solvent B over 90 minutes at a flow rate of $200 \mathrm{nl} / \mathrm{min}$. Mass spectrometry 
511 analysis was performed on a Q-Exactive plus mass spectrometer equipped with a

512 nanoelectrospray ion source (both Thermo Fisher Scientific) using a hybrid DDA (top5)/PRM

513 LC-MS analysis. In detail, each MS1 scan was followed by high-collision-dissociation (HCD) of

514 the precursor masses of the imported isolation list and the 5 most abundant precursor ions

515 with dynamic exclusion for 20 seconds. For each mutant and protease, a specific LC-MS

516 method was generated. Total cycle time was approximately 1 second. For MS1, 3e6 ions were

517 accumulated in the Orbitrap cell over a maximum time of $100 \mathrm{~ms}$ and scanned at a resolution

518 of 70,000 FWHM (at $200 \mathrm{~m} / \mathrm{z}$ ). Targeted MS2 scans were acquired at a target setting of $3 \mathrm{e} 6$

519 ions, accumulation time of $100 \mathrm{~ms}$ and a resolution of 35,000 FWHM (at $200 \mathrm{~m} / \mathrm{z}$ ) and a mass

520 isolation window to 0.4 Th. MS1 triggered MS2 scans were acquired at a target setting of 1e5

521 ions, a resolution of 17,500 FWHM (at $200 \mathrm{~m} / \mathrm{z}$ ) and a mass isolation window of 1.4 Th. Singly

522 charged ions and ions with unassigned charge state were excluded from triggering MS2 events.

523 The normalized collision energy was set to $27 \%$ and one microscan was acquired for each

524 spectrum. The acquired raw-files were converted to mgf-file format using MSConvert (v 3.0,

525 proteowizard) and searched using MASCOT (Matrix Science, Version: 2.4.1) against a decoy

526 database containing normal and reverse sequences of the predicted SwissProt entries of

527 Staphylococcus aureus (strain NCTC 8325 / PS 47, www.ebi.ac.uk, release date 2020/08/21).

528 The 5 LtaA mutants and commonly observed contaminants (in total 6,574 sequences) were

529 generated using the SequenceReverser tool from the MaxQuant software (Version 1.0.13.13).

530 The search criteria were set as following: full tryptic specificity was required (cleavage after

531 lysine or arginine residues); 3 missed cleavages were allowed; carbamidomethylation (C) was

532 set as fixed modification and oxidation (M) as variable modification. The mass tolerance was

533 set to $10 \mathrm{ppm}$ for precursor ions and 0.02 Da for fragment ions. Then, Scaffold (version

534 Scaffold_4.11.1, Proteome Software Inc., Portland, OR) was used to validate MS/MS based

535 peptide and protein identifications. Peptide identifications were accepted if they could be

536 established at a probability greater than $97.0 \%$ by the Scaffold Local FDR algorithm. Protein

537 identifications were accepted if they could be established at a probability higher than $99.0 \%$

538 to achieve an FDR less than $1.0 \%$ and contained at least 2 identified peptides. Protein

539 probabilities were assigned by the Protein Prophet algorithm [57]. Proteins that contained

540 similar peptides and could not be differentiated based on MS/MS analysis alone were grouped

541 to satisfy the principles of parsimony. Subsequently, all raw-files were imported into Skyline

542 for protein/peptide quantification. To control for variation in sample amounts, all intensities 
were normalized against the 4 cysteine-free reference peptides. Only peptides that could be confidently identified by database searching were considered for quantification by PRM using the predicted transitions. Statistical analysis and ratio calculations to compare the relative abundance of the peptides between non-crosslinked and crosslinked peptides were performed in Excel. Histograms and P values were generated using Prism 9.

Docking of glycolipid. A 1,2-dihexadecanoic-3-O-( $\beta$-D-glucopyranosyl-1-6-O- $\beta$-Dglucopyranosyl-sn-Glycerol molecule was docked to the LtaA inward-facing model with Autodock Vina [58]. The initial glycolipid coordinates were generated from 2D geometry in Phenix (eLBOW) [59]. The stereochemistry was corrected in Phenix (REEL)[59]. Docking was performed over a search space of $50 \times 44 \times 76 \AA^{3}$ covering the central cavity.

S. aureus phenotypic complementation assay. Generation of pLOW-ItaA and of Staphylococcus aureus NCTC8325 $\Delta$ /taA genotype was previously described [26]. pLOW vector was used for construction of ItaA complentary strains. Point mutations were generated by extension overlap PCR, and then with restriction-ligation cloning using Sall and Notl cloned into pLOW vector [60]. For cloning purposes E. coli IM08B was used[61]. The sequence of the resulting constructs was confirmed by DNA sequencing (Microsynth). After conformation of the correct constructs, pLOW vector carrying ItaA WT or point mutations were introduced into S. aureus NCTC8325 $\Delta /$ taA by electrophoresis with erythromycin selection $(5 \mu \mathrm{g} / \mathrm{ml})$. S. aureus cells were grown in $3 \mathrm{ml}$ of Luria-Bertani (LB) medium at $37^{\circ} \mathrm{C}$ with $200 \mathrm{rpm}$ until OD600 of 0.3. For complementary strains containing a pLOW vector, a final concentration of $5 \mu \mathrm{g} / \mathrm{ml}$ was added to the medium. For the serial dilutions, $5 \mu \mathrm{l}$ of the original and its dilutions were spotted on LB agar plates buffered with sodium phosphate at $\mathrm{pH} 6.4$ complemented with 0.1 mM IPTG. The plates were incubated overnight at $37^{\circ} \mathrm{C}$. Pictures were taken the next morning.

Preparation of S. aureus membranes for LC-MS analysis. S. aureus cells were grown in $3 \mathrm{ml}$ LB medium at $37{ }^{\circ} \mathrm{C}$ with $200 \mathrm{rpm}$ until $\mathrm{OD}_{600}$ of 0.4 . For complementary strains containing a $571 \mathrm{pLOW}$ vector, a final concentration of $5 \mu \mathrm{g} / \mathrm{ml}$ and $0.1 \mathrm{mM}$ IPTG were added to the medium. 572 After harvesting the cells were resuspended in $10 \mathrm{mM}$ Tris $\mathrm{pH} 8.0 ; 1 \mathrm{mM}$ EDTA; $25 \mu \mathrm{g} / \mathrm{ml}$ 573 Iysostaphin, and incubated for $0.5 \mathrm{~h}$ at $37^{\circ} \mathrm{C}$. Cells were further subjected to sonication, 574 followed by collection of membranes by ultracentrifugation. The membranes were 
resuspended in $100 \mathrm{mM}$ Tris- $\mathrm{HCl} ; 5 \%$ SDS; $10 \mathrm{mM}$ tris(2-carboxyethyl) phosphine (TCEP). Samples were sonicated for 10 minutes, followed by shaking for $1 \mathrm{~h}$ at $37^{\circ} \mathrm{C}$ with $500 \mathrm{rpm}$. To reduce and alkylate the disulfides a final concentration of $15 \mathrm{mM}$ iodoacetamide was added, and the samples were incubated for $0.5 \mathrm{~h}$ in the dark at room temperature. Samples were loaded on S-trap Micro Spin column (Protifi). After washing, on column peptide digestion was performed by addition of trypsin in $50 \mathrm{mM}$ triethylammonium bicarbonate (TEAB) buffer, and incubation of $1 \mathrm{~h}$ at $47{ }^{\circ} \mathrm{C}$. Digested peptides were collected by passing $50 \mathrm{mM}$ triethylammonium bicarbonate (TEAB) buffer, $0.2 \%$ formic acid $(w / v)$ in distilled water, and $0.2 \%$ formic acid $(\mathrm{w} / \mathrm{v})$ in $50 \%$ acetonitrile $(\mathrm{v} / \mathrm{v})$ through the column and dried in a SpeedVac (Labconco). Dried peptides were re-suspended in $0.1 \%$ formic acid $(\mathrm{w} / \mathrm{v})$ and stored at $-20^{\circ} \mathrm{C}$.

Targeted PRM LC-MS analysis of LtaA WT and mutants. As a first step, PRM assays [55] for all possible peptides of LtaA with a length of 6 to 25 amino acids comprising double- and triplecharged precursor ions were created. Five peptides were identified to match the length and charge criteria, leading to ten PRM assays in total. These were used to identify LtaA membrane fractions of wild-type $S$. aureus. The setup of the $\mu$ RPLC-MC system was previously described[56]. Mass spectrometry analysis was conducted using a Q-Exactive mass spectrometer with a nano-electrospray ion source (both Thermo Fisher Scientifice). Each MS1 scan was followed by high-collision-dissociation (HCD) of the ten LtaA precursor ions in PRM

594 mode using a global isolation mass list. By applying strict identification criteria, three peptide ions of LtaA LTNYNTRPVK (2+ and 3+ ion) and MQDSSLNNYANHK (2+) were identified, and these were used for label-free PRM quantification. To control for protein variation between different samples, the total ion chromatography (only comprising peptide with two or more charges) was determined for each sample by label-free quantification using Progenesis QI (version 2.0, Waters) and then used to normalize the samples. The integrated peak areas of

600 the three peptide ions that were quantified by PRM were summed up, and used for LtaA quantification.

Modeling of inward-facing conformation. The inward-facing conformation was modelled under the assumption of inverted repeats [47]. Sequence alignments between the two repeats of each domain of LtaA were performed. We structurally aligned R1D1 (residues 16105) with R2D1 (residues 109-189), and R1D2 (residues 220-302) with R2D2 (residues 309- 
393) using the structure alignment program TMalign resulting in two pairs of alignments.

608 These two pairs of alignments were then used together to build up the final pair-wise 609 alignment between the LtaA sequence and a template in which the LtaA sequence repeats were rearranged in the order R1D2-R1D1-R2D2-R2D1. The initial sequence alignment was

611 then refined by removing gaps in the transmembrane regions and in the secondary structure 612 elements. Further refinements were made to match the secondary structure as observed in 613 the outward-open crystal structure. In particular, we aimed to maintain the helical regions in 614 the template where possible, subject to the pseudo-symmetry between the two MFS 615 transporter domains. We used this alignment and the X-ray crystal structure of LtaA (PDB entry 6S7V)[26] to construct the inward-facing model templates using Modeller 9v24. In 100

617 templates, the side chain packing of the models were re-built using SCWRL4 model. Next, we 618 selected 7 models with the highest MODELLER score and the best MolProbity [62] profile for further analysis. Then, we repacked the side chains using SCWRL4.0[63] and as a last step the models were energetically minimized after placing them in the lipid bilayer using the Gromacs 2019.6 steepest descent algorithm for 5,000 steps [64]. To further validate the quality of the models, we assessed the stereochemistry. Evaluation of the model using MolProbity showed that the final minimized models have reasonable qualities (MolProbity score: 2.00-2.3, Ramachandran favored: 92.1-93.6\%, and Ramachandran outliers: 0.8-1.90\%) (Suppl. Table 1).

Molecular dynamics simulations of inward-facing conformation models. To study their dynamics, each of the optimized inward-facing models was placed in a heterogenous lipid bilayer (POPG (65\%), diacylglycerol (20\%), cardiolipin (10\%), and gentiobiosyl-diacylglycerol (5\%)) and then solvated in TIP3P water with $150 \mathrm{mM} \mathrm{NaCl}$. The all-atom CHARMM36m force field was used for lipids, ions, and protein [65-67]. All simulations were performed using GROMACS 2019.6 [64]. The starting systems were energy-minimized for 5,000 steepest descent steps and equilibrated first for 1 ns of MD simulations in a canonical (NVT) ensemble and then for $7.5 \mathrm{~ns}$ in an isothermal-isobaric (NPT) ensemble under periodic boundary 634 conditions. The initial restrains on the positions of nonhydrogen protein atoms were 4,000 $635 \mathrm{~kJ} \cdot \mathrm{mol}^{-1} \cdot \mathrm{nm}^{2}$. During equilibration, these restraints were gradually released. Particle-mesh 636 Ewald summation with cubic interpolation and a 0.12-nm grid spacing was used to treat long637 range electrostatic interactions [68]. The time step was initially $1 \mathrm{fs}$, and was then increased 638 to 2 fs during the NPT equilibration. The LINCS algorithm was used to fix all bond lengths [69]. 
Constant temperature was set with a Berendsen thermostat [70], combined with a coupling constant of $1.0 \mathrm{ps}$. A semi-isotropic Berendsen barostat [70] was used to maintain a pressure of 1 bar. During production runs, the Berendsen thermostat and barostat were replaced by a Nosé-Hoover thermostat [71] and a Parrinello-Rahman barostat [72] The unconstrained production trajectories were analyzed with Visual Molecular Dynamics (VMD) [73] and MDAnalysis package $[74,75]$. A simulation of each inward-facing model was performed for 150 ns.

Molecular dynamics simulations of outward-facing conformation. The outward-facing structure of LtaA (PDB ID 6S7V) was embedded in a lipid bilayer composed of POPG-DAG-CLgentiobiosyl-diacylglycerol using CHARMM-GUI [76]. The system was then solvated in TIP3P water with $150 \mathrm{mM} \mathrm{NaCl}$. The all-atom CHARMM36m force field was used for lipids, ions, and protein [65-67]. All simulations were performed using GROMACS 2019.6 [64]. Simulations were performed with similar protocols as described above for inward-facing models. The simulation of the outward-facing structure was performed for $960 \mathrm{~ns}$.

\section{$\underline{\text { References }}$}

656 1. Wang, S.C., et al., Expansion of the Major Facilitator Superfamily (MFS) to include novel 657 transporters as well as transmembrane-acting enzymes. Biochimica Et Biophysica Acta-Biomembranes, 658 2020. 1862(9).

659 2. Quistgaard, E.M., et al., Understanding transport by the major facilitator superfamily (MFS): 660 structures pave the way. Nat Rev Mol Cell Biol, 2016. 17(2): p. 123-32.

661 3. Reddy, V.S., et al., The major facilitator superfamily (MFS) revisited. FEBS J, 2012. 279(11): $\mathrm{p}$. 662 2022-35.

663 4. Drew, D., et al., Structures and General Transport Mechanisms by the Major Facilitator 664 Superfamily (MFS). Chem Rev, 2021. 121(9): p. 5289-5335.

665 5. Ben-Zvi, A., et al., Mfsd2a is critical for the formation and function of the blood-brain barrier. 666 Nature, 2014. 509(7501): p. 507-11.

667 6. Nguyen, L.N., et al., Mfsd2a is a transporter for the essential omega-3 fatty acid 668 docosahexaenoic acid. Nature, 2014. 509(7501): p. 503-6.

669 7. Harvat, E.M., et al., Lysophospholipid flipping across the Escherichia coli inner membrane 670 catalyzed by a transporter (Lp/T) belonging to the major facilitator superfamily. J Biol Chem, 2005. 671 280(12): p. 12028-34.

672 8. Abramson, J., et al., Structure and mechanism of the lactose permease of Escherichia coli. 673 Science, 2003. 301(5633): p. 610-5.

6749 9. Debruycker, V., et al., An embedded lipid in the multidrug transporter LmrP suggests a 675 mechanism for polyspecificity. Nature Structural \& Molecular Biology, 2020. 27(9): p. 829-835.

676 10. Heng, J., et al., Substrate-bound structure of the E. coli multidrug resistance transporter MdfA. 677 Cell Res, 2015. 25(9): p. 1060-73.

678 11. Deng, D., et al., Molecular basis of ligand recognition and transport by glucose transporters. 679 Nature, 2015. 526(7573): p. 391-6. 
12. Deng, D., et al., Crystal structure of the human glucose transporter GLUT1. Nature, 2014. 510(7503): p. 121-5.

13. Nomura, N., et al., Structure and mechanism of the mammalian fructose transporter GLUT5. Nature, 2015. 526(7573): p. 397-401.

14. Sun, L., et al., Crystal structure of a bacterial homologue of glucose transporters GLUT1-4. Nature, 2012. 490(7420): p. 361-6.

15. Billesbølle, C.B., et al., Structure of hepcidin-bound ferroportin reveals iron homeostatic mechanisms. Nature, 2020. 586(7831): p. 807-811.

688 16. Pan, Y., et al., Structural basis of ion transport and inhibition in ferroportin. Nature 689 Communications, 2020. 11(1): p. 5686.

690 17. Qureshi, A.A., et al., The molecular basis for sugar import in malaria parasites. Nature, 2020. 691 578(7794): p. 321-325.

692 18. Lin, Y., et al., A dual substrate-accessing mechanism of a major facilitator superfamily protein facilitates lysophospholipid flipping across the cell membrane. J Biol Chem, 2018. 293(51): p. 1991919931.

19. Angers, M., et al., Mfsd2a encodes a novel major facilitator superfamily domain-containing protein highly induced in brown adipose tissue during fasting and adaptive thermogenesis. Biochem J, 2008. 416(3): p. 347-55. dependent Lysophosphatidylcholine Transporter MFSD2A. J Biol Chem, 2016. 291(18): p. 9383-94.

21. Cater, R.J., et al., Structural basis of omega-3 fatty acid transport across the blood-brain barrier. Nature, 2021.

22. Kawahara, A., et al., The sphingolipid transporter spns2 functions in migration of zebrafish myocardial precursors. Science, 2009. 323(5913): p. 524-7.

704 23. Zhu, X., et al., Biological function of SPNS2: From zebrafish to human. Mol Immunol, 2018. 103: 705 p. 55-62.

706 24. Vu, T.M., et al., Mfsd2b is essential for the sphingosine-1-phosphate export in erythrocytes and 707 platelets. Nature, 2017. 550(7677): p. 524-528.

708 25. Grundling, A. and O. Schneewind, Genes required for glycolipid synthesis and lipoteichoic acid 709 anchoring in Staphylococcus aureus. J Bacteriol, 2007. 189(6): p. 2521-30.

710 26. Zhang, B., et al., Structure of a proton-dependent lipid transporter involved in lipoteichoic acids 711 biosynthesis. Nat Struct Mol Biol, 2020. 27(6): p. 561-569.

712 27. Parlet, C.P., M.M. Brown, and A.R. Horswill, Commensa Staphycocci influence Staphylococcus aureus SKin Colorizatin and Disease. Trends in Microbiology, 2019. 27(6): p. 497-507.

28. Sakr, A., et al., Staphylococcus aureus Nasal Colonization: An Update on Mechanisms, Epidemiology, Risk Factors, and Subsequent Infections. Front Microbiol, 2018. 9: p. 2419.

29. Ahn, K.B., et al., Lipoteichoic Acid Inhibits Staphylococcus aureus Biofilm Formation. Front Microbiol, 2018. 9: p. 327.

30. Hesser, A.R., et al., The length of lipoteichoic acid polymers controls Staphylococcus aureus cell size and envelope integrity. J Bacteriol, 2020.

31. Xia, G., T. Kohler, and A. Peschel, The wall teichoic acid and lipoteichoic acid polymers of Staphylococcus aureus. Int J Med Microbiol, 2010. 300(2-3): p. 148-54.

32. Percy, M.G. and A. Grundling, Lipoteichoic acid synthesis and function in gram-positive bacteria. Annu Rev Microbiol, 2014. 68: p. 81-100.

33. Fischer, W., et al., Structural Requirements of Lipoteichoic Acid Carrier for Recognition by the Poly(Ribitol Phosphate) Polymerase from Staphylococcus-Aureus $H$ - a Study of Various Lipoteichoic Acids, Derivatives, and Related-Compounds. Journal of Biological Chemistry, 1980. 255(10): p. 45504556.

$728 \quad 34 . \quad$ Neumann, J., D. Rose-Sperling, and U.A. Hellmich, Diverse relations between $A B C$ transporters and lipids: An overview. Biochim Biophys Acta Biomembr, 2017. 1859(4): p. 605-618. 
35. Pomorski, T.G. and A.K. Menon, Lipid somersaults: Uncovering the mechanisms of proteinmediated lipid flipping. Prog Lipid Res, 2016. 64: p. 69-84.

36. Perez, C., et al., Structure and mechanism of an active lipid-linked oligosaccharide flippase. Nature, 2015. 524(7566): p. 433-8.

37. Brunner, J.D., et al., X-ray structure of a calcium-activated TMEM16 lipid scramblase. Nature, 2014. 516(7530): p. 207-12. 38. Hiraizumi, M., et al., Cryo-EM structures capture the transport cycle of the P4-ATPase flippase. Science, 2019. 365(6458): p. 1149-1155. 39. Timcenko, M., et al., Structure and autoregulation of a P4-ATPase lipid flippase. Nature, 2019. 571(7765): p. 366-370.

740 40. Mi, W., et al., Structural basis of MsbA-mediated lipopolysaccharide transport. Nature, 2017. 549(7671): p. 233-237.

41. Menon, I., et al., Opsin is a phospholipid flippase. Curr Biol, 2011. 21(2): p. 149-53.

42. Pomorski, T. and A.K. Menon, Lipid flippases and their biological functions. Cell Mol Life Sci, 2006. 63(24): p. 2908-21.

43. Olsen, J.A., et al., Structure of the human lipid exporter $A B C B 4$ in a lipid environment. Nat Struct Mol Biol, 2020. 27(1): p. 62-70.

44. Kim, Y. and J. Chen, Molecular structure of human P-glycoprotein in the ATP-bound, outwardfacing conformation. Science, 2018. 359(6378): p. 915-919.

45. Perez, C., et al., Structure of Outward-Facing PglK and Molecular Dynamics of Lipid-Linked Oligosaccharide Recognition and Translocation. Structure, 2019. 27(4): p. 669-678.e5.

46. Timcenko, M., et al., Structural Basis of Substrate-Independent Phosphorylation in a P4-ATPase Lipid Flippase. J Mol Biol, 2021: p. 167062.

47. Radestock, S. and L.R. Forrest, The alternating-access mechanism of MFS transporters arises from inverted-topology repeats. J Mol Biol, 2011. 407(5): p. 698-715.

48. Basilio, D., et al., Conformational changes required for $\mathrm{H}(+) / \mathrm{Cl}(-)$ exchange mediated by a $\mathrm{CLC}$ transporter. Nat Struct Mol Biol, 2014. 21(5): p. 456-63.

49. $\mathrm{Bi}, \mathrm{Y}$., et al., Architecture of a channel-forming O-antigen polysaccharide ABC transporter. Nature, 2018. 553(7688): p. 361-365.

50. Koch, H.U., R. Haas, and W. Fischer, The role of lipoteichoic acid biosynthesis in membrane lipid metabolism of growing Staphylococcus aureus. Eur J Biochem, 1984. 138(2): p. 357-63.

51. Jardetzky, O., Simple allosteric model for membrane pumps. Nature, 1966. 211(5052): p. 96970.

52. Zhang, B. and C. Perez, Stabilization and Crystallization of a Membrane Protein Involved in Lipid Transport. Methods Mol Biol, 2020. 2127: p. 283-292.

53. Jorasch, P., et al., A UDP glucosyltransferase from Bacillus subtilis successively transfers up to four glucose residues to 1,2-diacylglycerol: expression of ypfP in Escherichia coli and structural analysis of its reaction products. Mol Microbiol, 1998. 29(2): p. 419-30.

54. Kiriukhin, M.Y., et al., Biosynthesis of the glycolipid anchor in lipoteichoic acid of Staphylococcus aureus RN4220: Role of YpfP, the diglucosyldiacylglycerol synthase. Journal of Bacteriology, 2001. 183(11): p. 3506-3514.

55. Peterson, A.C., et al., Parallel reaction monitoring for high resolution and high mass accuracy quantitative, targeted proteomics. Mol Cell Proteomics, 2012. 11(11): p. 1475-88.

56. Ahrne, E., et al., Evaluation and Improvement of Quantification Accuracy in Isobaric Mass TagBased Protein Quantification Experiments. J Proteome Res, 2016. 15(8): p. 2537-47.

57. Nesvizhskii, A.l., et al., A statistical model for identifying proteins by tandem mass spectrometry. Anal Chem, 2003. 75(17): p. 4646-58.

58. Trott, O. and A.J. Olson, AutoDock Vina: improving the speed and accuracy of docking with a new scoring function, efficient optimization, and multithreading. J Comput Chem, 2010. 31(2): p. 45561. 
59. Adams, P.D., et al., PHENIX: a comprehensive Python-based system for macromolecular structure solution. Acta Crystallogr D Biol Crystallogr, 2010. 66(Pt 2): p. 213-21.

60. Heckman, K.L. and L.R. Pease, Gene splicing and mutagenesis by PCR-driven overlap extension. Nat Protoc, 2007. 2(4): p. 924-32.

61. Monk, I.R., et al., Complete Bypass of Restriction Systems for Major Staphylococcus aureus Lineages. mBio, 2015. 6(3): p. e00308-15.

62. Chen, V.B., et al., MolProbity: all-atom structure validation for macromolecular crystallography. Acta Crystallogr D Biol Crystallogr, 2010. 66(Pt 1): p. 12-21.

63. Krivov, G.G., M.V. Shapovalov, and R.L. Dunbrack, Jr., Improved prediction of protein side-chain conformations with SCWRL4. Proteins, 2009. 77(4): p. 778-95.

64. Abraham, M.J., et al., GROMACS: High performance molecular simulations through multi-level parallelism from laptops to supercomputers. SoftwareX, 2015. 1-2: p. 19-25.

65. Best, R.B., et al., Optimization of the Additive CHARMM All-Atom Protein Force Field Targeting Improved Sampling of the Backbone $\phi, \psi$ and Side-Chain $\chi 1$ and $\chi 2$ Dihedral Angles. Journal of Chemical Theory and Computation, 2012. 8(9): p. 3257-3273.

66. Jorgensen, W.L., et al., Comparison of simple potential functions for simulating liquid water. The Journal of Chemical Physics, 1983. 79(2): p. 926-935.

67. Klauda, J.B., et al., Update of the CHARMM All-Atom Additive Force Field for Lipids: Validation on Six Lipid Types. The Journal of Physical Chemistry B, 2010. 114(23): p. 7830-7843.

68. Darden, T., D. York, and L. Pedersen, Particle mesh Ewald: An N.log $(N)$ method for Ewald sums in large systems. The Journal of Chemical Physics, 1993. 98(12): p. 10089-10092.

69. Hess, B., et al., LINCS: A linear constraint solver for molecular simulations. Journal of Computational Chemistry, 1997. 18(12): p. 1463-1472.

70. Berendsen, H.J.C., et al., Molecular dynamics with coupling to an external bath. The Journal of Chemical Physics, 1984. 81(8): p. 3684-3690.

71. Hoover, W.G., Canonical dynamics: Equilibrium phase-space distributions. Phys Rev A Gen Phys, 1985. 31(3): p. 1695-1697.

72. Parrinello, M. and A. Rahman, Polymorphic transitions in single crystals: A new molecular dynamics method. Journal of Applied Physics, 1981. 52(12): p. 7182-7190.

73. Humphrey, W., A. Dalke, and K. Schulten, VMD: Visual molecular dynamics. Journal of Molecular Graphics, 1996. 14(1): p. 33-38.

74. Gowers, R., et al., MDAnalysis: A Python Package for the Rapid Analysis of Molecular Dynamics Simulations. 2016. 98-105.

75. Michaud-Agrawal, N., et al., MDAnalysis: A toolkit for the analysis of molecular dynamics simulations. Journal of Computational Chemistry, 2011. 32(10): p. 2319-2327.

76. Wu, E.L., et al., CHARMM-GUI Membrane Builder toward realistic biological membrane simulations. J Comput Chem, 2014. 35(27): p. 1997-2004. 


\section{Acknowledgments}

828 We thank Prof. Jan-Willem Veening for providing us the S. aureus NCTC8325, S. aureus

829 NCTC8325 $\Delta /$ taA, E. coli IMO8B cells and the pLOW-vector. We thank Xiaochun Li Blatter for 830 assistance in cell expression and membranes preparations. This work was supported by the 831 Swiss National Science Foundation (SNSF) (PP00P3_198903 to C.P), the Helmut Horten 832 Stiftung (HHS) (to C.P), and by the Max Planck Society and the German Research Foundation 833 (SFB 807: Membrane Transport and Communication, to A.R.M and G.H). E.L. was funded by 834 the Biozentrum International PhD Program and the HHS.

835

\section{Author Contributions}

837 E.L. performed in vitro and in vivo biochemical characterization of LtaA and variants. A.R.M. 838 performed computational analysis. C.P. supervised the biochemical analysis. G.H. supervised 839 computational analysis. A.S. and E.L. performed mass spectrometry analysis. E.L., A.R.M, and 840 C.P analyzed the computational, structural and functional data. C.P. conceived and directed 841 the project. All authors contributed to manuscript writing and revision.

842

\section{Author Information}

844 Competing interests: None declared. 


\section{Figures and Figure legends}

A

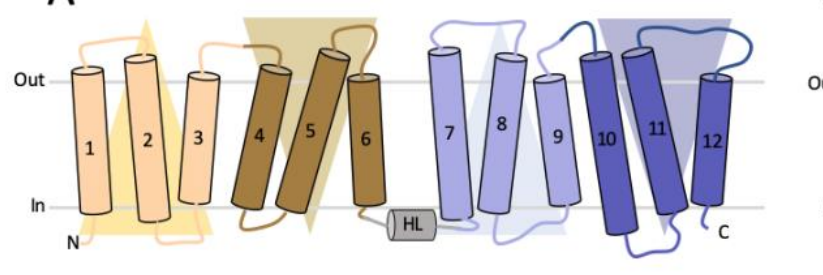

B

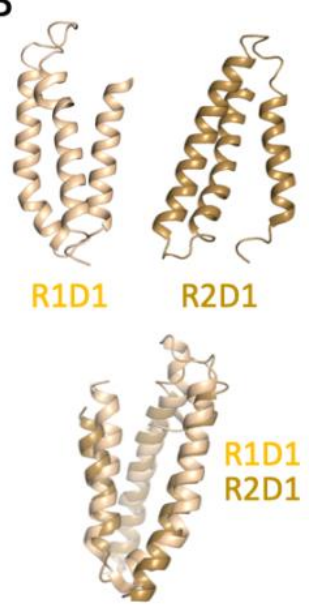

C

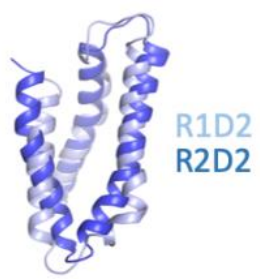

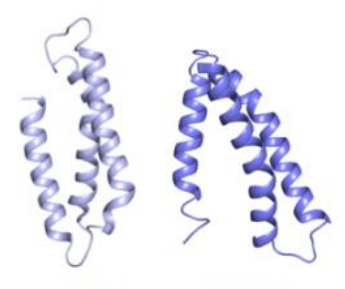

R1D2 R2D2
860
C TM8 TM5

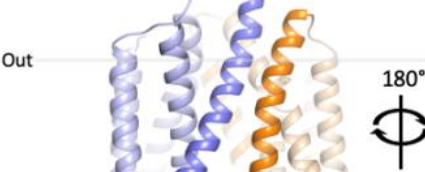

9
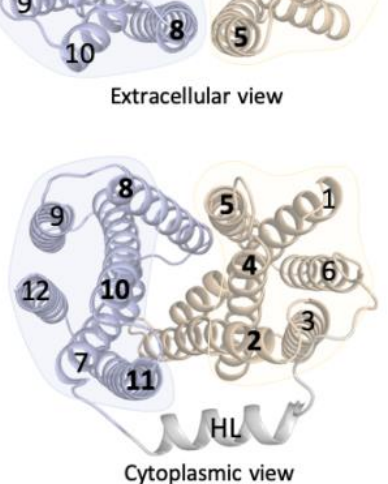

Figure 1. 'Repeat swap' modeling of inward-facing LtaA. A. Topology representation of LtaA. Domain-1 (N-terminal) and domain-2 (C-terminal) are shown in light orange and light blue, respectively. B. Individual repeat domains as observed in outward-facing LtaA (PDB ID 6S7V), and superposition of inverted repeats (r.m.s.d. = $2.5 \AA$ and $3.0 \AA$ for aligned Ca atoms of R1D1/R2D1 and R1D2/R2D2, respectively). R1D1 and R2D1 indicate the first and second repeats in the N-terminal domain, respectively, whereas R1D2 and R2D2 indicate the first and second repeat in the C-terminal domain, respectively. Colors are according to panel A. C. Sideviews of inward-facing LtaA model showing TM helices that line the lateral openings. Extracellular and cytoplasmic views are also shown. 
A
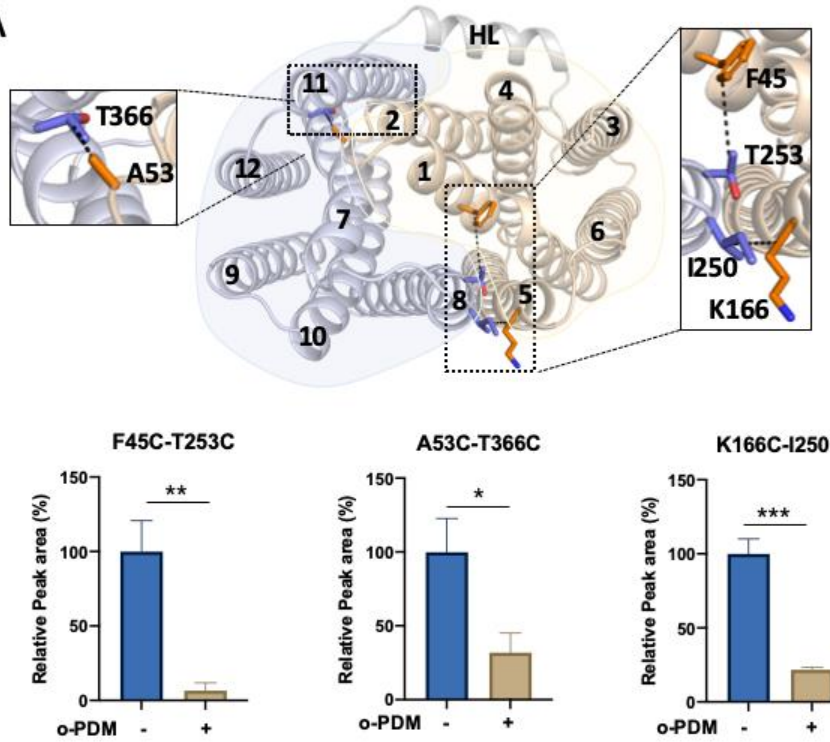

A53C-T366C

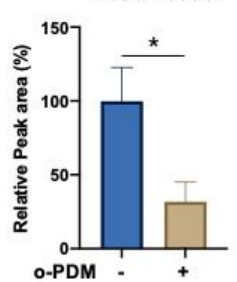

B

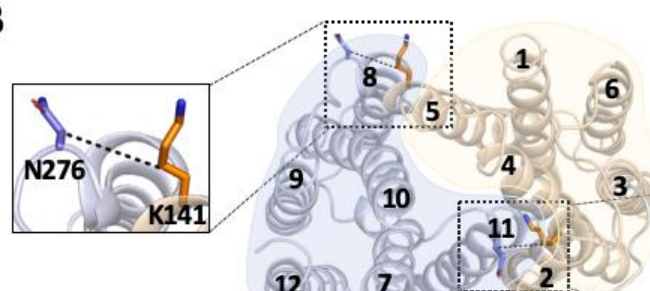

(12) 7

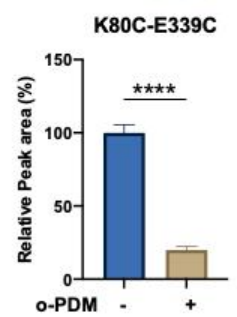

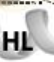
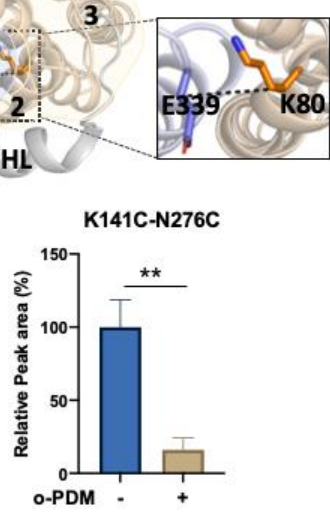

C
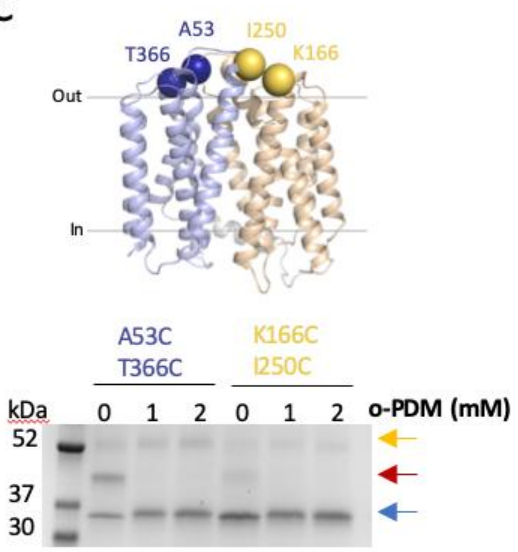

30
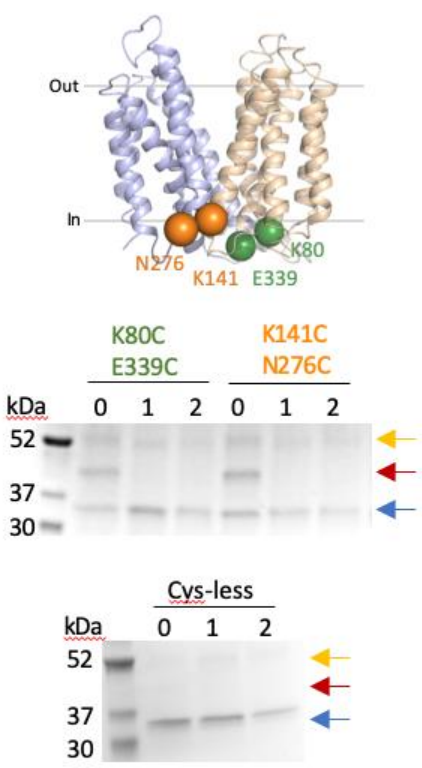

Figure 2. LtaA adopts inward- and outward-facing states. Selected residues for cross-linking of LtaA in inward-facing conformation (A) and outward-facing conformation (B). N-terminal and C-terminal domains are shown in light orange and light blue, respectively. The relative abundance of cysteine containing peptides in absence (-) or presence (+) of o-PDM is shown in histograms. Collision-induced dissociation (CID) spectrum of cysteine containing peptides and elution profiles of peptide fragments are shown in supplementary figure 3 . Error bars

883 indicate standard deviation (s.d.), $\mathrm{n} \geq 3 .{ }^{*}: \mathrm{P} \leq 0.05, * *$ : $\mathrm{P} \leq 0.01, * * *$ : $\mathrm{P} \leq 0.001, * * * *$ : $\mathrm{P} \leq 0.0001 . \mathrm{C}$.

884 Cross-linking analysis of LtaA in proteoliposomes. Positions of selected cysteine pairs at the 885 extracellular and cytoplasmic regions of LtaA are shown as spheres. SDS-PAGE show band shifts of samples treated with mPEG5K after irreversible cross-link with o-PDM. Separated species are indicated with arrows. Blue arrow: non-PEGylated LtaA; red arrow: PEGylated LtaA; orange arrow: LtaA dimer. 
A

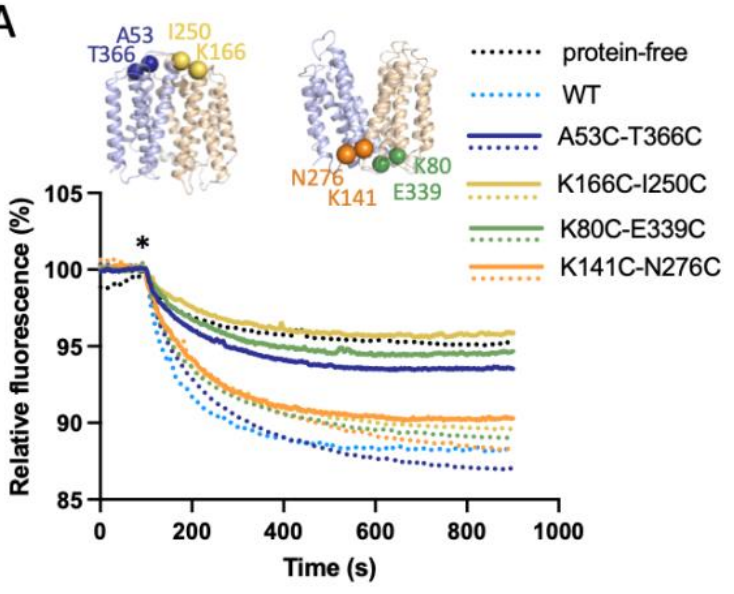

C

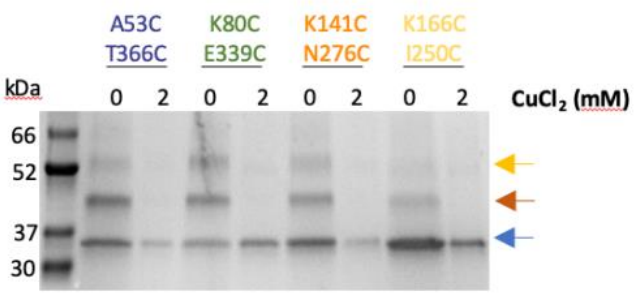

B

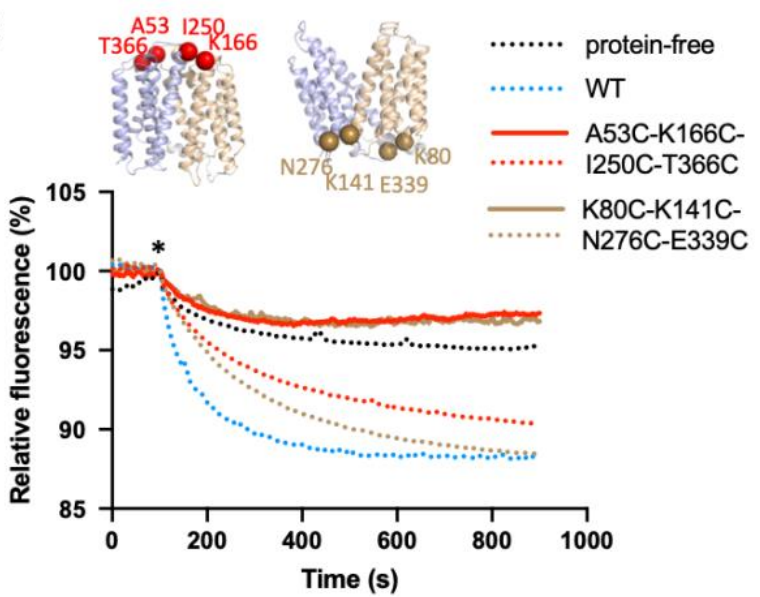

D

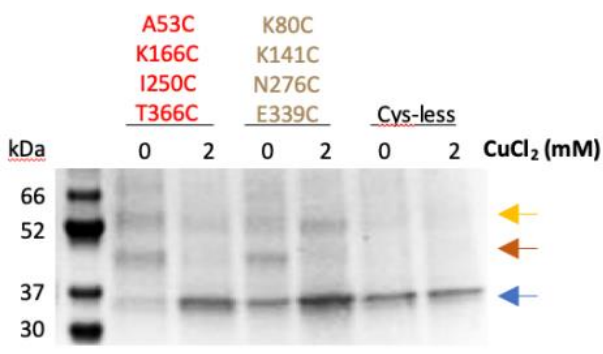

Figure 3. Cycling through outward- and inward-facing conformations is essential for LtaA activity. A,B. Proton-transport activity of LtaA and variants after chemical crosslinking with $\mathrm{CuCl}_{2}$ (solid lines) or in absence of cross-linking treatment (dotted lines) $(n \geq 3)$. Proteoliposomes and protein-free liposomes containing $100 \mathrm{mM} \mathrm{KCl}$ were diluted in buffer containing $10 \mathrm{mM} \mathrm{KCl}, 90 \mathrm{mM} \mathrm{NaCl}$ and $\mathrm{ACMA} . \mathrm{H}^{+}$influx was initiated by establishing a membrane potential upon addition of the potassium ionophore valinomycin (asterisk). C.D. 
A
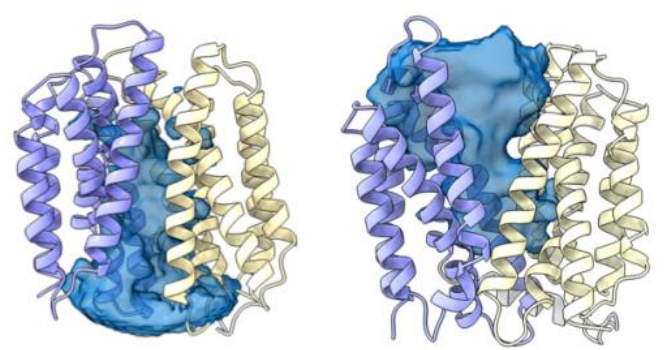

C

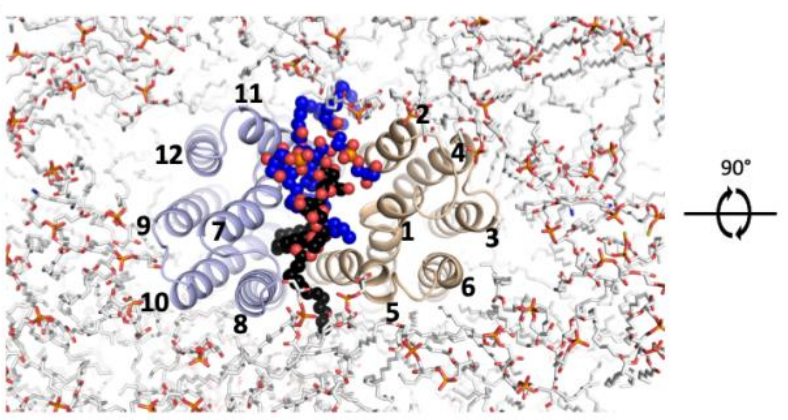
in light orange and light blue, respectively.
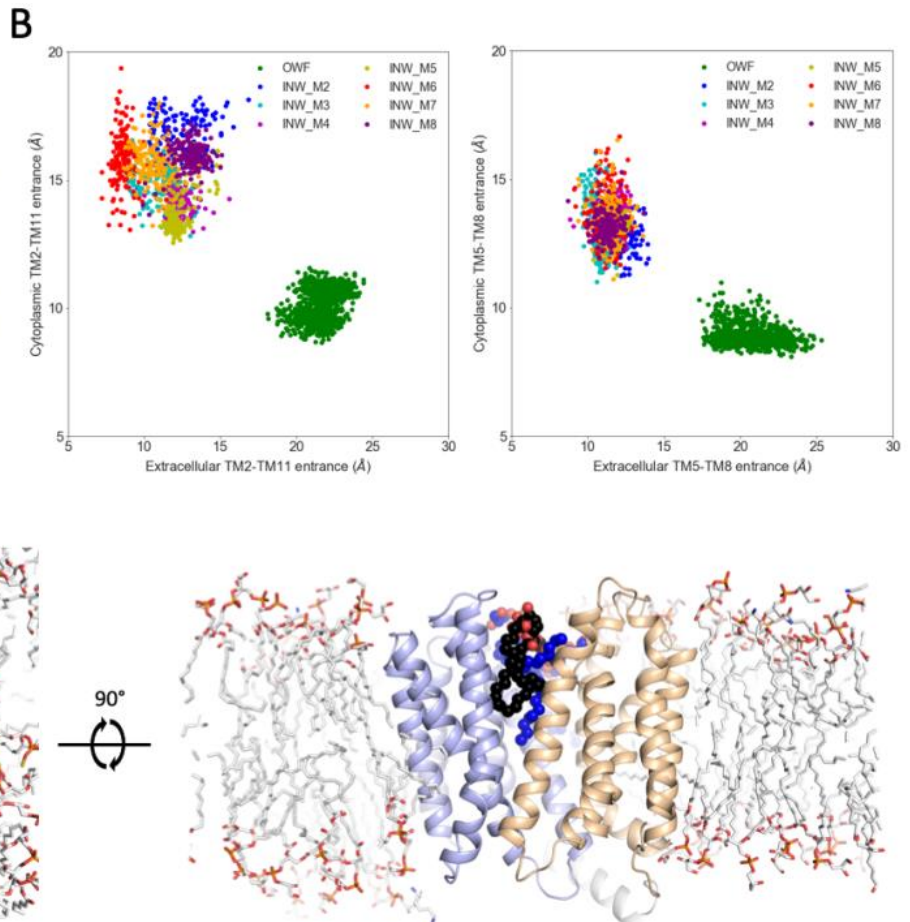

Figure 4. LtaA displays asymmetric opening of cavities and lateral 'gates'. A. Representative view of solvent exposed cavity of inward-facing and outward-facing LtaA as observed during MD simulations. B. Analysis of distances between TM helices lining the cytoplasmic and extracellular lateral openings of outward-facing and inward-facing models. The center of masses of the C $\alpha$ atoms of extracellular residues 52-57 (TM2), 163-167 (TM5), 250-255 (TM8), 364-367 (TM11), and of cytoplasmic residues 77-81 (TM2), 139-143 (TM5), 273-276 (TM8), 341-344 (TM11), were used for the calculation of inter-TM distances. C. Intrusion of gentiobiosyl-diacylglycerol (black spheres) and POPG (blue spheres) molecules in the extracellular cavity of LtaA during simulations. $\mathrm{N}$-terminal and $\mathrm{C}$-terminal domains are shown 

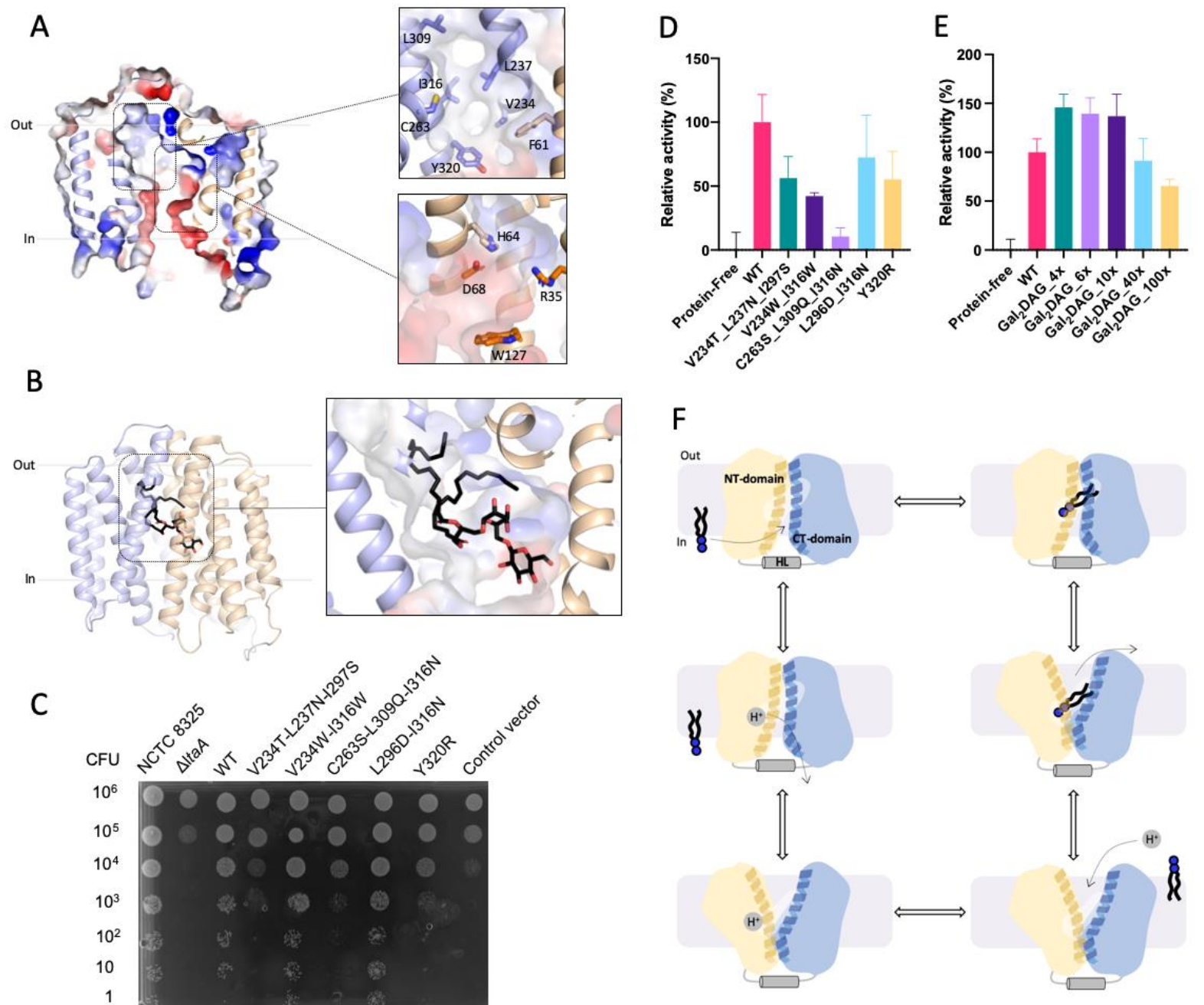

\section{C}

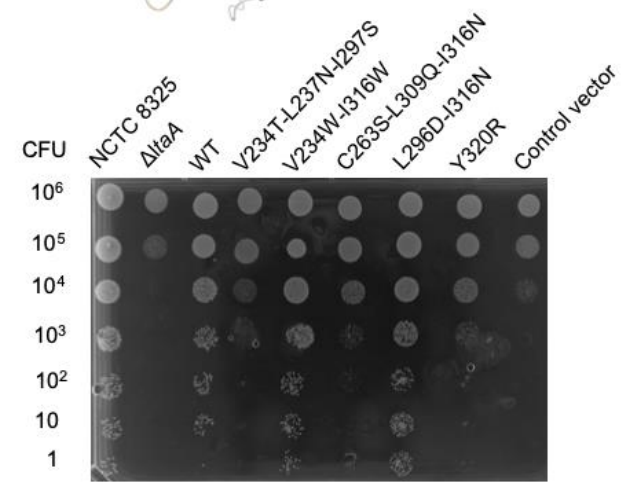

929 Figure 5. Hydrophilic and hydrophobic cavities participate in 'trap-and-flip' of lipids. A.

930 Vacuum electrostatic surface representation of inward-facing model of LtaA. Residues

931 forming the hydrophobic and hydrophilic pockets are shown. B. A model of a glycolipid

932 molecule docked into the amphipathic cavity of LtaA. The lipid tail length corresponds to C16

933 chains. N-terminal and C-terminal domains are shown in light orange and light blue,

934 respectively. C. S. aureus cell growth on LB agar plates containing 0.1 mM IPTG, buffered at $935 \mathrm{pH}$ 6.4. The $\triangle /$ taA mutant is complemented with pLOW vector carrying a ItaA-WT gene or the 936 annotated point mutations; Control vector indicates the pLOW vector carrying an unrelated 937 gene (dCas9). D. Mutagenesis analysis of the hydrophobic pocket. Relative flipping activity of 938 LtaA-WT and variants. Error bars show s.d. of technical replicates, $n \geq 3$. E. Headgroup 939 selectivity analysis. Relative flipping activity of $L$ taA in the presence of different concentrations 940 of digalactosyl-diacylglycerol ( $\mathrm{Gal}_{2} \mathrm{DAG}$ ). Molar excess of $\mathrm{Gal}_{2} \mathrm{DAG}$ over $\mathrm{Glc}_{2}$-DAG-NBD is 941 indicated. Error bars show s.d. of technical replicates, $n \geq 3$. F. Proposed mechanism of LtaA 
bioRxiv preprint doi: https://doi.org/10.1101/2021.08.05.453402; this version posted August 5,2021 . The copyright holder for this preprint (which was not certified by peer review) is the author/funder, who has granted bioRxiv a license to display the preprint in perpetuity. It is made available under aCC-BY-ND 4.0 International license.

942 catalyzed glycolipid transport. Schematic of conformational states throughout LtaA transport

943 cycle. 